Dermatologische Zeitschrift. 1936;73:I-IV

\title{
Contents, Vol. 73, 1936
}

Inhaltsverzeichnis.

Orig $\cdot$ inalarbeiten.

Seit $\beta$

Arning, Ed., Zur Geburt der Anthrarobinti $\pi$ ktur 8

Bode, H. G., Über Nahbestrahlung von Epitheliomen

Brambíila, S., Zum Problem unseres Verhaltens beím Auftrete $\pi$ von Herpes

Zoster unter spezifischer Syphilistherapie 264

Engelhardt, W. und F. Sträter, Zur Frage der Begutachtung Schmierõlgeschädigter 125

Epstein, Stephan, Riesenzellen-Sarkom auf Lupus vulgaris 66

Epstein, Stephan und Max Jeßner, Zur Solganaltherapie von Lupus erythematodes, Tuberkuliden und Akne 1

Fink, M. I. siehe Lewin, J. M.

Halter, Klaus, Zur Pathogenese der Chondrodermatitis nodularis chron.

helicis 270

Hoffmann, Erich, Über ein einfaches Verfahren zur Darstellung der epi-

dermoídalen Anhänge der Haut (Haarfollikel, Talg- und Schweißdrüsen)

im Verlauf ihrer Entwicklung (Mazerationsmethode fotaler Haut). . 185 Hruszek, H., Über

gleichzeitiges Vorkommen von Tuberculosis verrucosa

cutis und Lichen ruber verrucosus 71

Jeßner, Max siehe Epstein, Stephan.

Jordan, A., Bericht über 673 Fãlle von Acne vulgaris

Jordan, A. und B. Bydníck, Hyperkeratosis subungualis congenita oder

Pachyonychia congenita $\quad 326$

Lewin, J. M, Über Färbung der Hautnarbe $\pi$ bei den Arbeitern der Kohleni $\pi$ dustrie $\quad 135$

- M. M. Lewin und M. I. Fink, Zur Diagnostik der Heilung der Gonorrhoe 201

Lewin, M. M. siehe Lewin, J. M.

Maschkilleisson, L. N. und L. A. Neradow, Über Lupus erythematodes

familiaris 9

Neradoiv, L. A. siehe Maschkilleisson, L. N.

Oberreit, E., Über ampulläre Phlebektasien in halbseítîger Anordnung . . 75

Bydnick, B. siehe Jordan, A.

Schaaf, Fritz, Neue Ergebnisse der Melaninforschung 14

Schmidt, Werner, Statistisch-klinische Betrachtungen über die von 1926 bis 1935 beobachteten Erkrankungen an Lues I und Lues II 285

Schreus, H. Th., Über das serologische Geschehen bei Lues, Gonorrhoe und Tuberkulose nebst einer Auswertung an 1000 Seren in bezug auf die Übereinstimmu $\pi \mathrm{g}$ des klinischen und serologischen Befundes 
Sellei, Josef, Beitrãge zur Pathogenese und Therapie der Dermatomyositis 197

Sträter, F. siehe Engelhardt, W.

Werner, Fritz, Über einen Fall von Varizellen, nach mehrfachem Auftreten

von Herpes zoster 204

Wieämann, Albert, Beitrãge zur Pathologie der Gef äßerkrankungen der Haut 241 , 329

Sammelreferate.

Allgemeine Pathologie und pathologische Anatomie. Von Dr. med. habil.

Franz ifoe $7 / 8$-Tübingen 35

Dermatitis, Ekzem und andere Allergosen. (Überbliek über das Jahr 1935.)

Von J. A. Folpmers-Gronmgen 80

Pathophysiologie und Stoîfwechsel (Herbst 1934 bis Herbst 1935). Von Prof.

Dr. C. Moncorps-Monchen 137, 207

IV

Inhaltsverzeichnis.

S $\theta$ it $\theta$

Erytheme, Herpesgruppe, Pemphigusgruppe. Von Dr. Albin Proppe-Diissel-

dorf 147

Infektionskrankheiten der Haut (außer Tuberkulose und Lepra):

I. Dermatomykosen. Von Prof. Dr. P. W. Schmidt-Freiburg i. Br. . 220

Pyodermien, Diphtherie, Milzbrand. Von Ferdinand John-Freiburg i. Br 229

Vakzine, Variola, Alastrim, Melkerknoten, Scharlach, Masern, Vari-zellen, Noma, Sodoku,

Zoonosen. Von Ferdinand Jo/m-Freiburg i. Br. 232

Tuberkulose (1935/36). Von Prof. 0. Bittrich-Breslau 293

Lepra. Von Prof. Dr. V. Klingmüller-Kiel 298

Gummöse Mykosen und verwandte Krankheiten (Sporotríchose, Blasto-

mykose usw.). Von Dr. W. Gasper-Kiel 308

Psoriasis, Lichenerkrankungen, Verhornungsanomalien, Pigmentanomalien,

Nagel- und Haarerkrankungen, Erkrankungen der Talgdrüsen und

Schweißdrüsen (Anfang 1935 bis Anfang 1936). Von Dr. H. 0. Loos-

Innsbruck $\quad 340$

Zusammenfassende Arbéiten diagnostischen und therapeutischen Inhalts.

Ubersícht fiber dermato-venerologische Arbéiten aus Zeitschriften der USSR.

Erschienen vom 1. Juii 1934 bis 1. Juli 1935. Von A. Jordan-

Moskau 105, 161

Gesellschaftsberichte.

Trühjahrstagung der Vereinigung rheinisch-westfälischer Dermatologen in

Düsseldorf. Sitzung vom 19. Mai 1935 44

Royal Society of Medicine. Section of Dermatology. Sitzung vom 17. Mai 193452

Sitzung vom 21. Juni 1934; Sitzung vom 5. Juli 1935

168

Sitzung vom 17. Oktober 1935

Sitzung vom 19. Dezember $1935 \quad 367$

North of England Dermatological Society. Sitzung vom 12. April 1935 in

Sheffield; Sitzung vom 10. Mai 1935 in Liverpool; Sitzung vom

21. Juni 1935 in Leeds .172

North British Dermatological Society. Sitzung vom 21. März 1935 in Glasgow . 119

- $\quad$ Sitzung vom 20. Juni 1935 in Dundee; Sitzung vom 11. Oktober 1935 
in Manchester 175

Société Française de Dermatologie et de Syphiligraphie. Sitzung vom

4. April 1935

54

Sitzung vom 9. Mai 1935

176

Sitzung vom 6. Juni 1935

235

Buchbesprechungen 58, 120, 179, 239, 314, 369

50 Jahre Gram'sche Färbung 59

Albert Jesionek $\dagger$

122

Personalien und Tagesnachrichten $\quad 59,123,183,240,316,342$ 